\title{
Challenges of Downstream Processing for the Production of Biodiesel from Microalgae
}

\author{
Vânia Novais Pôjo \\ Faculdade de Engenharia, Universidade do Porto, Rua Dr. Roberto Frias 4200-465 PORTO \\ (vaniapojo@gmail.com)
}

\begin{abstract}
The continuous reliance on fossil fuels is unsustainable, due to the depletion of global reserves and the greenhouse gas emissions associated with their use. Therefore, there are vigorous research initiatives intended to develop renewable alternatives. Microalgae are a promising alternative for biodiesel production and have received increasing attention during the last few decades. However, is not yet sufficiently cost-effective to compete with petroleum-based conventional fuels. This happens essentially because of downstream processing - harvesting microalgae biomass and extraction of lipids are two of the most expensive processes from the overall process. Harvesting, drying, cell disruption, oil extraction and transesterification (into biodiesel) are highlighted processes in this review article. The techniques associated with each process present advantages and handicaps that are here discussed. Improvements that will directly affect the final production costs of microalgal biomass-based biofuels are also proposed.
\end{abstract}

Subject Headings. Bioenergy, Biotechnology, Bioprocess

Author Keywords. Microalgae, Downstream Processing, Biodiesel

\section{Introduction}

The rapid growth of world's population and rise of developing countries has led to increased energy needs (Harun, Danquah, and Forde 2010). This is the cause of nowadays scarcity of petrochemical resources as well as environmental pollution problems. These are two critical challenges that need to be addressed by our society. In a result of this, many countries set national biofuel production targets and provided incentives and support to accelerate the growth of the bioenergy industry. For example, in the United States, Renewable Fuels Standard (RFS), part of the Energy Independence and Security Act (EISA) of 2007, set the annual production goal of $\approx 136$ billion litres typically of biofuels until 2022 (U.S. Congress 2007). To avoid adverse impacts on the supply of food for human consumption, EISA further specifies that 60 of the 136 billion liters of renewable fuels produced in 2022 should be advanced biofuels produced from algal biomass, for example. However, the current production capacity of advanced biofuels is less than 37 billion liters worldwide (Yue, You, and Snyder 2014).

Microalgae are leading the development of third generation of biofuels and have recently received much attention for the commercial production of advanced biofuels, including biodiesel. Despite the wide range of advantages associated with the production of biodiesel with these microorganisms (e.g. high carbon adsorption capacity (contributing to greenhouse gas (GHG)) reduction), an economic feasibility of the microalgae-based biofuels industry has not yet been achieved because it still doesn't have an highly economic process 
that integrates the multiple steps associated with the downstream process - harvest, extraction, and conversion of biomass to biodiesel (Mata, Martins, and Caetano 2012).

Downstream process represents $60 \%$ of the total biodiesel production cost. In this way, it is of great importance to reduce these costs. Harvesting of microalgae corresponds of $20 \%$ of the total production cost of biodiesel (varies according with the type of harvest technology used and the density of the microalgal culture (Mata, Martins, and Caetano 2010).

The oil extraction from dried biomass can be accomplished using various cell rupturing techniques including autoclave, ultrasound, homogenization, and bead milling. Organic solvents, acids, alkalis, or enzymes can also be used for the chemical or biological breakdown of the cell wall.

After the oil is extracted, it's converted into biodiesel through a transesterification reaction in methanol with an acid or an alkaline catalyst (normally alkaline). However, this reaction produces toxic chemicals and the recovery of the product isn't easy (Chisti 2007).

Despite the recognition of all the difficulties that downstream processes for the production of biodiesel offer, there is a tremendous potential to improve the economics of microalgal biofuels (Rawat et al. 2013).

This review discusses the techniques used for harvesting microalgae, extract the lipids, and convert them to biodiesel. It also provides perspectives on the most suitability technique according with its techno-economic feasibility.

\section{Downstream Processing}

An integrated production of biodiesel from microalgae (Fig. 1) includes a microalgal cultivation step, followed by the separation of the cells from the growth medium and subsequent lipid extraction for biodiesel production through transesterification.

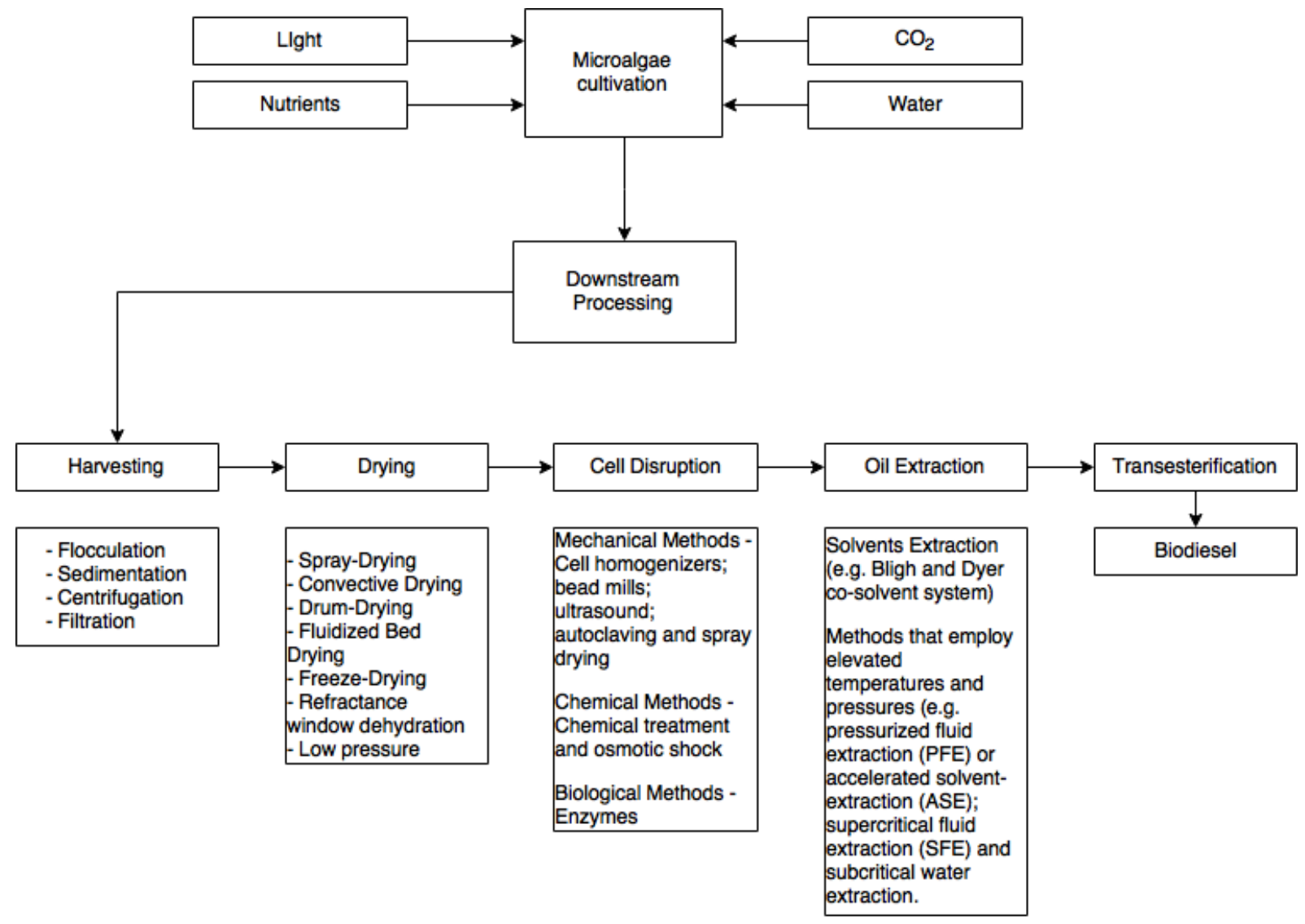

Figure 1: Integrated process for biodiesel production from microalgae 


\subsection{Cell Harvesting}

Harvesting is an extremely challenging process and in need for research, in order to develop an appropriate and economical system for any microalga species, since an universal harvesting method, does not exists (Uduman et al. 2010).

The main problems associated are (1) cells size, which is typically small ( 3 to $30 \mu \mathrm{m}$ ) making it difficult to separate them from de culture media (Mata, Martins, and Caetano 2012); (2) the cells normally carry negative charge and excess algogenic organic material prevent easily settling by gravity (Brennan and Owende 2010); (3) low concentrations of biomass found in the production systems (Mata, Martins, and Caetano 2012). All of this makes harvesting an expensive process.

Most common harvesting methods include sedimentation, centrifugation, filtration, ultrafiltration, sometimes with an additional flocculation step or with a combination of flocculation-flotation (Mata, Martins, and Caetano 2010).

Harvesting normally involves two processes: bulk harvesting, whose purpose is to separate biomass from the bulk suspension (e.g. through flocculation and flotation) and thickening, which follows the bulk harvesting and aims to concentrate the slurry (possible to use either centrifugation or filtration) (Golueke and Oswald 1965).

Selection of the most appropriate harvesting technique depends on the properties of the microalga at stake (density and size, respectively), as well as the specifications of the desired product (Golueke and Oswald 1965).

Flocculation is the first step in biomass recovery process and is intended to aggregate the microalgae, to facilitate and increase the efficiency of the processes that follow (Elmaleh et al. 1991). Since microalgae are negatively charged, there are repulsive forces that do not allow the cells to aggregate. However, this charge can be neutralized or reduced by the addition of chemicals, better known as flocculants.

Some researchers (Weissman and Goebel 1987) compared four primary harvesting methods for the purpose of biofuels production: microstraining, belt filtering, flotation with float collection, and sedimentation. All these methods make separation based on the size and density of microalgae. Microstrainers are available in large unit sizes have a mechanical simplicity and a low cost for large-sized microalgae. However, incomplete solids removal and difficulty in handling fluctuations of solid contents (Rossignol et al. 1999) as well as the need for an earlier flocculation process are problems associated with this approach.

Filter presses can be operated under pressure or vacuum and are used to recover large quantities of biomass but it can be a slow process and because of this, sometimes, unsatisfying.

Larger microalgae (as Coelastrum proboscideum and S. platensis) can be easily recover through filtration, however this process is not so suitable to species with smaller dimensions (as Scenedesmus, Dunaliella, or Chlorella) (Grima et al. 2003). Other filtration processes are also suitable for biomass recovery, such as microfiltration and ultrafiltration (especially for the recovery of fragile cells), however, these processes are more expensive because of the need for membrane replacement and pumping. Same thing happens when applying tangential flow filtration.

According to Richmond (2004), to select the most adequate harvesting procedure, it's very important to take into account the quality of the desired product. For low value products, gravity sedimentation may be used, possibly enhanced by flocculation. For high-value products (such as for food or aquaculture applications), it is often recommended to use 
continuously operating centrifuges that can process large volumes of biomass. Centrifuges concentrate biomass quickly and can be easily cleaned or sterilized to effectively avoid bacterial contamination or fouling of raw product. Against this process is the considerable cost associated.

In order to choose the proper harvesting method it is important to know which cell disruption technique will be used (next step of the overall process, if drying is dismissed), since the accepted moisture level will depend on it. (Richmond 2004; Grima et al. 2003; Amaro, Macedo, and Malcata 2012).

Taking into consideration the cost of thermal drying comparing to mechanical dewatering, a combination of methods can be used, such as, a pre-concentration with a mechanical dewatering step (microstrainer, filtration, or centrifugation) and then, a post-concentration (with a centrifuge or a thermal drying) (Mata, Martins, and Caetano 2010). The right is to apply first the processes leading to larger volume reductions, followed by those that are more selective (and also more expensive).

Another harvesting process is the electrolytic method, which is able to separate cells without chemicals addition. As a downside, the power that requires produces high temperatures that can destruct the system. Another disadvantage is the cathode fouling.

A potential alternative for efficient mass harvesting of microalgae is flotation, which uses an oxidant to destabilize suspended microalgal cells (Engler 1985).

\subsection{Biomass Drying}

In order for biodiesel production to be an economically feasible process, shortening the processes adjacent to its production is fundamental and, therefore, has been the starter for several investigations. According to some authors (Xu et al. 2011), the use of wet biomass for the subsequent processes gives rise to higher value biofuels, on the other hand the use of dry biomass gives a higher fossil energy ratio, which is defined as the ratio between the amount of energy that goes into the final fuel product (fuel energy output) and the amount of fossil energy input (non-renewable energy) required for fuel production.

Several methods can be used to dry biomass, such as spray-drying (Leach, Oliveira, and Morais 1998), convective drying (Desmorieux and Decaen 2005), drum-drying, fluidized bed drying, freeze-drying (Cordero and Voltolina 1997), refractance window dehydration technology (Nindo and Tang 2007), low-pressure shelf drying and sun-drying (Prakash et al. 1997). These processes can increase biomass shelf life.

The method that is more economically suitable for the biodiesel production would be the sun-drying, but due to the high content of water, the necessity for large drying surface and the possibility of losing some of the bioreactive products makes it ineffective (Li et al. 2008). As for the other techniques, although they're more efficient, they're not economically feasible for biodiesel (Mata, Martins, and Caetano 2010).

In order to choose the most suitable drying method or even decide to bypass it, will depend on the desired final product. Considering that biodiesel is a low value product, skipping the drying process can be a possibility, not neglecting that further studies will be needed.

\subsection{Cell Disruption}

Cell disruption is a very important step of the overall process because most microalgae have a strong cell-wall, which difficult the lipids extraction and reduces the yield obtained. To overcome this problem, several methods can be used to break the cell wall. These methods can be mechanical (e.g. cell homogenizers, bead mills, ultrasound, autoclaving and spray drying), chemical (comprise the use of chemical treatments and osmotic shock) and 
biological (involve the use of enzymes to degrade polysaccharides and/or proteins) (Kim et al. 2013; Mata, Martins, and Caetano 2012).

Ultrasound and microwave assisted extractions were reported by Cravotto et al. (2008) as being great to improve the extraction of bioactive substances from microalgae at shorter reaction times and up to 10 times lower solvent consumption. These authors also found that ultrasounds worked better than microwaves, improving higher yields of oil extraction. Additionally, they proved these methods to be less toxic, more economical and more efficient than the co-solvents extraction approaches.

Another authors (Burja et al. 2007) proved the efficiency of ultrasound combined with solvents (methanol:chloroform) for breaking the cell walls of Thraustochytrium sp. ONC-T18, concluding that this was the best technique to extract fatty acids, from all the evaluated ones.

Lee et al. (2010) tested autoclaving, bead-beating, microwaves and sonication to identify the most effective for lipids extraction from Botryococcus sp., Chlorella vulgaris and Scenedesmus sp.. They concluded that the most effective, simple and easy was microwave.

The efficiency of fatty acid extraction from Scenedesmus obliquus was assessed by Wiltshire et al. 2000, who compared a technique that included quartz sand, solvent and ultrasound (in freeze dried algal) with another technique combining solvents with subsequent incubation, concluding that the first one had twice the extraction efficiency and it is a method that conserves the fatty acids present in the biomass without affecting the products.

Another method referred (Sommerfeld et al. 2010) as being good at improving the lipid extraction efficiency is electroporation. This method alters the cell membranes and walls, reducing the duration of the extraction and the use of solvent without affecting the composition of the extracted fatty acids.

Bead-beating method has also been studied and proved to be better in lipids extraction of Botryococcus braunii, than sonication, homogenization, French pressing or lyophilization (Geciova, Bury, and Jelen 2002). However, this is not an easy to scale up method.

Given the above, microwave has been proved to be the most efficient, simple and easy to scale-up disruption cell method. Nonetheless, the choice of the technique to be used must take into account the species that will be used.

\subsection{Oil Extraction}

Microalgae possess two kinds of lipids - polar lipids and non-polar lipids. Polar lipids are associated with the membrane structure and flexibility (e.g. phospholipids and glycolipids). Non-polar lipids are the glycerides (mono, di and tri) and also some vitamins, hydrocarbons, was esters and sterols (Benemann and Oswald 1996).

The species of microalgae have a great role on the applicability of the extraction method and it's efficiency. For example microalgae that lack a cell wall are more prone to shear breakage than ones that contain a rigid cell wall. Other important aspects are the cell wall composition, strength and structure (when applicable) (Mata, Martins, and Caetano 2012).

The most recognized method for total lipid quantification is the Bligh and Dyer co-solvent system (Bligh and Dyer 1959; Grima et al. 1994). This technique consists in exposing the lipid-containing tissue to a miscible co-solvent mixture - methanol, chloroform and water in the ratios of 1:2:0.8 and 2:2:1.8 before and after dilution, respectively.

The homogenate obtained is then diluted in water, creating a biphasic system where the non-lipids accumulate on the water/methanol phase and the lipids accumulate on the 
chloroform phase. After isolating the chloroform, it is evaporated leaving an extract of purified lipid (Mata, Martins, and Caetano 2012).

The downsides of this method are that the chloroform can extract more than just lipids compromising the true lipid content (Iverson, Lang, and Cooper 2001); the toxicity of chloroform and methanol; as well as the difficulty to scale up.

There has been some research on the Bligh and Dyer method to mitigate its disadvantages. (Grima et al. 1994) compared seven solvent mixtures, concluding that a mixture of ethanol at $96 \%$ and hexane/ethanol at $96 \%$ resulted in higher lipids yield. Hara and Radin (1978) proposed the use of less toxic and cheaper solvents (hexane/isopropanol). Lewis, Nichols, and McMeekin (2000) concluded that adding to the biomass the solvents chloroform, methanol and then water, in this order of increasing polarity, the total amount of lipids and fatty acids extraction increased about $30 \%$. What happens is that the initial contact with the chloroform or with a mixture of chloroform and metanol, weakens the association between lipids and proteins in the cell membrane, making them permeable for water, to separate them.

Solvent extraction techniques still have some problems that need to be overcome, such as the difficulty to scale-up and the high quantity of solvents required for extraction (Benemann and Oswald 1996). In this way, some other options should be considered. Methods that employ elevated temperatures and pressures can be used. Some examples of these, are pressurized fluid extraction (PFE) (Denery et al. 2004) or accelerated solventextraction (ASE) (Richter et al. 1996), supercritical fluid extraction (SFE) (Cheung 1999) and subcritical water extraction (SCWE) (Eikani, Golmohammad, and Rowshanzamir 2007). All of these have the same advantage in common, which is the facility of access to the lipids extracted because of the higher temperature and pressure applied, that increases the solvation power of the solvents as well as their capacity to solubilize the lipids extracted. Despite this, these techniques are not suitable for large scale, since the organic solvents used are expensive and the methods require an energy expenditure (Cooney, Young, and Nagle 2009).

Richter et al. (1996) and Mulbry et al. (2009) used ASE for lipid-extraction. The first ones, reported a very short extraction time (less than $15 \mathrm{~min}$ ), without causing thermal degradation of temperature-sensitive compounds. The second group of authors tested the ASE with three solvents (chloroform/methanol, isopropanol/hexane and hexane) and compared it with Folch method, concluding that the ASE method yielded higher values for total oil content.

SFE is a less effective technique in extracting polar compounds from natural matrices because of supercritical $\mathrm{CO}_{2}$ low polarity. This implies the addition of co-solvents, which are highly polar compounds that when added in small amounts, can produce substantial changes in the solvent properties of pure supercritical $\mathrm{CO}_{2}$ (Herrero, Cifuentes, and Ibanez 2006). In the end of the process, the product can be easily separated from the solvent just by lowering the temperature and pressure to atmospheric conditions, where the fluid returns to its original gaseous state and the extracted product remains as a liquid or solid.

Various authors already tested SFE of lipids, in order to test its suitability. Canela et al. (2002) concluded that both the temperature and the pressure affected the extraction rate and the effect of the temperature prevailed over that of the pressure. The extracts were rich in essential fatty acids. 
The main disadvantage of SFE of lipids from microalgae is that it requires high pressure equipment, which is expensive and energy intensive, increasing the operating costs and limiting applicability in biofuels production from microalgae.

Lipids extraction based on the use of water at temperatures just below the critical temperature (between 100 and $374{ }^{\circ} \mathrm{C}$ ) and a pressure high enough to maintain the liquid state, is possible with SCWE. The advantages and obstacles of SCWE, as an effective method for the isolation of high-quality essential oils were discussed by Luque de Castro, JiménezCarmona, and Fernández-Pérez (1999). In this technique, the lipids are easily extracted, because when the temperature and pressure return to atmospheric, the water is no longer miscible with the lipids. The greatest advantage associated with this method is the possibility of being applied directly to harvested microalgae without need for the dewatering step. However, such as the other techniques discussed above, SCWE has the same constraints energy intensive process and difficulty to scale-up.

\subsection{Oil Transesterification into Biodiesel}

Biodiesel is a mixture of fatty acid alkyl esters obtained by transesterification of oils or fats, whose main components are triglycerides (90 - $98 \%$ ). A smaller fraction of mono- and diglycerides, free fatty acids, residual amounts of phospholipids, phosphatides, carotenes, tocopherols, sulphur compounds and water (Bozbas 2008).

Transesterification is a three reversible steps reaction: triglycerides are first converted to diglycerides, then to monoglycerides and finally to esters (biodiesel) and glycerol (byproduct). Then, it follows re-esterification with a short chain alcohol (usually methanol), which ensures high volatility. These reagents, alcohol and oil, are used in the presence of a catalyst (usually $\mathrm{NaOH}$ ) in a molar ratio of $6: 1$ to guarantee that the reaction is driven in the direction of methyl esters (towards biodiesel - despite theoretical molar ratio is 3:1) (Fukuda, Kondo, and Noda 2001). The relationship between the feedstock mass input and biodiesel mass output is about 1:1, which means that the quantity of oil used results in the same quantity of biodiesel produced (theoretically $1 \mathrm{~kg}$ of oils produces $1 \mathrm{~kg}$ of biodiesel) (Mata, Martins, and Caetano 2010).

Other catalysts can be used (instead of $\mathrm{NaOH}$ ), such as acids (Fukuda, Kondo, and Noda 2001) and lipase enzymes (Sharma, Chisti, and Banerjee 2001). However, alkali-catalyzed transesterification is much faster (about 4000 times) than the acid catalyzed reaction (Fukuda, Kondo, and Noda 2001). Although the use of lipases offers important advantages, is not currently feasible because of the high cost associated (Fukuda, Kondo, and Noda 2001).

In respect to the alcohols used, another ones can be utilized, in spite of methanol, however methanol is the cheapest. A consequence of transesterification reaction is soap formation, which causes yield loss. To prevent this, the oil and alcohol must be dry and the oil should have a minimum of free fatty acids. In the end, biodiesel is recovered by repeated washing with water to remove glycerol and methanol (Chisti 2007).

An alternative to transesterification process is in situ transesterification, which happens directly inside the biomass and facilitates directly conversion of fatty acids to their alkyl esters, reducing the processing costs, by eliminating the solvent extraction step and alleviating the need for biomass drying in harvesting. It's a very promising alternative because alcoholysis of oil while in the biomass matrix leads to biodiesel higher yields and waste production is also reduced (Ehimen, Sun, and Carrington 2010). 


\section{Conclusions}

Most of the companies that intend to produce biodiesel from microalgae economically in the next few years, don't have sufficient technical expertise to do it, because of the current limitations essentially related to microalgae harvesting and oil extraction processes. In this way, investment in technological development and technical expertise is needed before algal biodiesel is economically viable and can become a reality.

In this way, it is essential to develop new strategies and to incorporate positive elements of the existing approaches.

New approaches should be applicable to any microalgal species without significant differences in effectiveness and must be scalable to meet the challenge of commercial biodiesel production. One of the best solutions to achieve these goals may be the direct conversion of wet microalgal biomass to biodiesel (avoiding the drying step).

The techniques used for harvest, extraction, and conversion must be environmentally sustainable while being sufficiently inexpensive to make microalgal biofuels competitive with petroleum-based transportation fuels.

This work allowed to conclude that most of the techniques available for downstream processing require high energy consumption, which is critical from an economic and environmental point of view.

Furthermore, the choice of the techniques for harvesting and dewatering determines the subsequent downstream unit operations, including the methods to be used for lipid extraction and possibly the transesterification reaction.

\section{References}

Amaro, Helena M., Ângela C. Macedo, and F. Xavier Malcata. 2012. "Microalgae: An Alternative as Sustainable Source of Biofuels?" Energy 44 (1): 158-66. DOI: 10.1016/j.energy.2012.05.006.

Benemann, John R, and William J Oswald. 1996. "Systems and Economic Analysis of Microalgae Ponds for Conversion of $\mathrm{CO} 2$ to Biomass." Final Report to the Department of Energy, Pittsburgh Energy Technology Center, DOE/PC/93204-T5. DOI: 10.2172/493389.

Bligh, E. G., and W. J. Dyer. 1959. "A Rapid Method of Total Lipid Extraction and Purification." Canadian Journal of Biochemistry and Physiology 37.

Bozbas, Kahraman. 2008. "Biodiesel as an Alternative Motor Fuel: Production and Policies in the European Union." Renewable and Sustainable Energy Reviews 12 (2): 542-52. DOI: 10.1016/j.rser.2005.06.001.

Brennan, Liam, and Philip Owende. 2010. "Biofuels from microalgae-A Review of Technologies for Production, Processing, and Extractions of Biofuels and Co-Products." Renewable and Sustainable Energy Reviews 14 (2). Elsevier Ltd: 557-77. DOI: 10.1016/j.rser.2009.10.009.

Burja, Adam M., Roberto E. Armenta, Helia Radianingtyas, and Colin J. Barrow. 2007. "Evaluation of Fatty Acid Extraction Methods for Thraustochytrium Sp. ONC-T18." Journal of Agricultural and Food Chemistry 55 (12): 4795-4801. DOI: 10.1021/jf070412s.

Canela, A. Paula R. F., Paulo T. V. Rosa, Marcia O. M. Marques, and M. Angela A. Meireles. 2002. "Supercritical Fluid Extraction of Fatty Acids and Carotenoids from the Microalgae Spirulina Maxima." Industrial \& Engineering Chemistry Research 41 (12): 3012-18. DOI: 10.1021/ie010469i. 
Cheung, P. 1999. "Temperature and Pressure Effects on Supercritical Carbon Dioxide Extraction of N-3 Fatty Acids from Red Seaweed." Food Chemistry 65 (3): 399-403. DOI: 10.1016/S0308-8146(98)00210-6.

Chisti, Y. 2007. "Biodiesel from Microalgae." Biotechnology Advances 25 (3). Elsevier Inc.: 294-306. DOI: 10.1016/j.biotechadv.2007.02.001.

Cooney, Michael, Greg Young, and Nick Nagle. 2009. "Extraction of Bio-oils from Microalgae." Separation \& Purification Reviews 38 (4): 291-325. DOI: 10.1080/15422110903327919.

Cordero, Beatriz, and Domenico Voltolina. 1997. "Viability of Mass Algal Cultures Preserved by Freezing and Freeze-Drying." Aquacultural Engineering 16 (4): 205-11. DOI: 10.1016/S0144-8609(97)00001-0.

Cravotto, Giancarlo, Luisa Boffa, Stefano Mantegna, Patrizia Perego, Milvio Avogadro, and Pedro Cintas. 2008. "Improved Extraction of Vegetable Oils under High-Intensity Ultrasound And/or Microwaves." Ultrasonics Sonochemistry 15 (5): 898-902. DOI: 10.1016/j.ultsonch.2007.10.009.

Denery, Judith R, Klaus Dragull, C.S Tang, and Qing X Li. 2004. "Pressurized Fluid Extraction of Carotenoids from Haematococcus Pluvialis and Dunaliella Salina and Kavalactones from Piper Methysticum." Analytica Chimica Acta 501 (2): 175-81. DOI: 10.1016/j.aca.2003.09.026.

Desmorieux, Hélène, and Nadège Decaen. 2005. "Convective Drying of Spirulina in Thin Layer." Journal of Food Engineering 66 (4): 497-503. DOI: 10.1016/j.jfoodeng.2004.04.021.

Ehimen, E.A., Z.F. Sun, and C.G. Carrington. 2010. "Variables Affecting the in Situ Transesterification of Microalgae Lipids." Fuel 89 (3): 677-84. DOI: 10.1016/j.fuel.2009.10.011.

Eikani, Mohammad H., Fereshteh Golmohammad, and Soosan Rowshanzamir. 2007. "Subcritical Water Extraction of Essential Oils from Coriander Seeds (Coriandrum Sativum L.)." Journal of Food Engineering 80 (2): 735-40. DOI: 10.1016/j.jfoodeng.2006.05.015.

Elmaleh, S, J Coma, A Grasmick, and L Bourgade. 1991. "Magnesium Induced Algal Flocculation in a Fluidized Bed." Water Science \& Technology 23 (7-9): 1695-1702.

Engler, C. R. 1985. "Disruption of Microbial Cells." In Comprehensive Biotechnology, edited by M Moo-Yoong, 305-24. Oxford: Pergamon Press.

Fukuda, Hideki, Akihiko Kondo, and Hideo Noda. 2001. "Biodiesel Fuel Production by Transesterification of Oils." Journal of Bioscience and Bioengineering. DOI: 10.1016/S1389-1723(01)80288-7.

Geciova, Jana, Dean Bury, and Paul Jelen. 2002. "Methods for Disruption of Microbial Cells for Potential Use in the Dairy Industry-a Review." International Dairy Journal 12 (6): 541-53. DOI: 10.1016/S0958-6946(02)00038-9.

Golueke, C G, and W J Oswald. 1965. "Harvesting and Processing Sewage-Grown Planktonic Algae." Journal Water Pollution Control Federation 37 (4): 471-98. http://www.jstor.org/stable/25035278\%0D.

Grima, E. M., E.-H Belarbi, F.G.A Fernández, A. R. Medina, and Yusuf Chisti. 2003. "Recovery of Microalgal Biomass and Metabolites: Process Options and Economics." Biotechnology Advances 20 (7-8): 491-515. DOI: 10.1016/S0734-9750(02)00050-2. 
Grima, E M, A R Medina, A G Giménez, J A Sánchez Pérez, F G Camacho, and J L García Sánchez. 1994. "Comparison between Extraction of Lipids and Fatty Acids from Microalgal Biomass." Journal of the American Oil Chemists' Society 71 (9): 955-59. DOI: 10.1007/bf02542261.

Hara, Atsushi, and Norman S. Radin. 1978. "Lipid Extraction of Tissues with a Low-Toxicity Solvent." Analytical Biochemistry 90 (1): 420-26. DOI: 10.1016/0003-2697(78)90046-5.

Harun, Razif, Michael K. Danquah, and Gareth M. Forde. 2010. "Microalgal Biomass as a Fermentation Feedstock for Bioethanol Production." Journal of Chemical Technology and Biotechnology 85: 199-203. DOI: 10.1002/jctb.2287.

Herrero, M, A Cifuentes, and E Ibanez. 2006. "Sub- and Supercritical Fluid Extraction of Functional Ingredients from Different Natural Sources: Plants, Food-by-Products, Algae and microalgaeA Review." Food Chemistry 98 (1): 136-48. DOI: 10.1016/j.foodchem.2005.05.058.

Iverson, Sara J., Shelley L. C. Lang, and Margaret H. Cooper. 2001. "Comparison of the Bligh and Dyer and Folch Methods for Total Lipid Determination in a Broad Range of Marine Tissue." Lipids 36 (11): 1283-87. DOI: 10.1007/s11745-001-0843-0.

Kim, Jungmin, Gursong Yoo, Hansol Lee, Juntaek Lim, Kyochan Kim, Chul Woong Kim, Min S. Park, and Ji Won Yang. 2013. "Methods of Downstream Processing for the Production of Biodiesel from Microalgae." Biotechnology Advances 31 (6): 862-76. DOI: 10.1016/j.biotechadv.2013.04.006.

Leach, G., G. Oliveira, and R. Morais. 1998. "Spray-Drying of Dunaliella Salina to Produce a $\beta$ Carotene Rich Powder."

Lee, Jae-Yon, Chan Yoo, So-Young Jun, Chi-Yong Ahn, and Hee-Mock Oh. 2010. "Comparison of Several Methods for Effective Lipid Extraction from Microalgae." Bioresource Technology 101 (1): S75-77. DOI: 10.1016/j.biortech.2009.03.058.

Lewis, T, P. D. Nichols, and T. A. McMeekin. 2000. "Evaluation of Extraction Methods for Recovery of Fatty Acids from Lipid-Producing Microheterotrophs." Journal of Microbiological Methods 43 (2): 107-16.

Li, Yanqun, Yanqun Li, Mark Horsman, Mark Horsman, Nan Wu, Nan Wu, Christopher Q Lan, Christopher Q Lan, Nathalie Dubois-Calero, and Nathalie Dubois-Calero. 2008. "Biofuels from Microalgae." Biotechnology Progress 24: 815-20. DOI: 10.1021/bp.070371k.

Luque de Castro, M.D, M.M Jiménez-Carmona, and V Fernández-Pérez. 1999. "Towards More Rational Techniques for the Isolation of Valuable Essential Oils from Plants." TrAC Trends in Analytical Chemistry 18 (11): 708-16. DOI: 10.1016/S0165-9936(99)00177-6.

Mata, T. M., A. A. Martins, and N. S. Caetano. 2010. "Microalgae for Biodiesel Production and Other Applications: A Review." Renewable and Sustainable Energy Reviews 14 (1): 217-32. DOI: 10.1016/j.rser.2009.07.020.

- - . 2012. "Microalgae Processing for Biodiesel Production." Advances in Biodiesel Production: Processes and Technologies, 204-31. DOI: 10.1016/B978-0-85709-1178.50009-5.

Mulbry, Walter, Shannon Kondrad, Jeffrey Buyer, and Devanand L. Luthria. 2009. "Optimization of an Oil Extraction Process for Algae from the Treatment of Manure Effluent." Journal of the American Oil Chemists' Society 86 (9): 909-15. DOI: 10.1007/s11746-009-1432-1. 
Nindo, C. I., and J. Tang. 2007. "Refractance Window Dehydration Technology: A Novel Contact Drying Method." Drying Technology 25 (1): 37-48. DOI: 10.1080/07373930601152673.

Prakash, J., B. Pushparaj, P. Carlozzi, G. Torzillo, E. Montaini, and R. Materassi. 1997. "Microalgal Biomass Drying by a Simple Solar Device." International Journal of Solar Energy 18 (4): 303-11. DOI: 10.1080/01425919708914325.

Rawat, I., R. Ranjith Kumar, T. Mutanda, and F. Bux. 2013. "Biodiesel from Microalgae: A Critical Evaluation from Laboratory to Large Scale Production." Applied Energy. DOI: 10.1016/j.apenergy.2012.10.004.

Richmond, A. 2004. Handbook of Microalgal Culture: Biotechnology and Applied Phycology. Blackwell Science Ltd. DOI: 10.1006/abio.2001.5321.

Richter, Bruce E., Brian A. Jones, John L. Ezzell, Nathan L. Porter, Nebojsa Avdalovic, and Chris Pohl. 1996. "Accelerated Solvent Extraction: A Technique for Sample Preparation." Analytical Chemistry 68 (6): 1033-39. DOI: 10.1021/ac9508199.

Rossignol, N., L. Vandanjon, P. Jaouen, and F. Quéméneur. 1999. “Membrane Technology for the Continuous Separation Microalgae/culture Medium: Compared Performances of Cross-Flow Microfiltration and Ultrafiltration." Aquacultural Engineering 20 (3): 191-208. DOI: 10.1016/S0144-8609(99)00018-7.

Sharma, Rohit, Yusuf Chisti, and Uttam Chand Banerjee. 2001. "Production, Purification, Characterization, and Applications of Lipases." Biotechnology Advances. DOI: 10.1016/S0734-9750(01)00086-6.

Sommerfeld, M, W Chen, Q Hu, D Giorgi, and T Navapanich. 2010. "Application of Electroporation for Lipid Extraction from Microalgae."

U.S. Congress. 2007. "Energy Independence and Security Act of 2007." Public Law, 110-40. http://scholar.google.com/scholar?hl=en\&btnG=Search\&q=intitle:ENERGY+INDEPENDEN CE+AND+SECURITY+ACT+OF+2007+An+Act\#0.

Uduman, Nyomi, Ying Qi, Michael K. Danquah, Gareth M. Forde, and Andrew Hoadley. 2010. "Dewatering of Microalgal Cultures: A Major Bottleneck to Algae-Based Fuels." Journal of Renewable and Sustainable Energy 2 (1): 12701. DOI: 10.1063/1.3294480.

Weissman, J.C., and R.P. Goebel. 1987. "Design and Analysis of Microalgal Open Pond Systems for the Purpose of Producing Fuels: A Subcontract Report." Golden, CO. DOI: 10.2172/6546458.

Wiltshire, Karen H., Maarten Boersma, Anita Möller, and Heinke Buhtz. 2000. "Extraction of Pigments and Fatty Acids from the Green Alga Scenedesmus Obliquus (Chlorophyceae)." Aquatic Ecology 34 (2): 119-26. DOI: 10.1023/A:1009911418606.

Xu, Lixian, Derk W.F. (Wim) Brilman, Jan A.M. Withag, Gerrit Brem, and Sascha Kersten. 2011. "Assessment of a Dry and a Wet Route for the Production of Biofuels from Microalgae: Energy Balance Analysis." Bioresource Technology 102 (8): 5113-22. DOI: 10.1016/j.biortech.2011.01.066.

Yue, Dajun, Fengqi You, and Seth W. Snyder. 2014. "Biomass-to-Bioenergy and Biofuel Supply Chain Optimization: Overview, Key Issues and Challenges." Computers and Chemical Engineering 66: 36-56. DOI: 10.1016/j.compchemeng.2013.11.016. 